\title{
IMMANANTS OF TOTALLY POSITIVE MATRICES ARE NONNEGATIVE
}

\author{
JOHN R. STEMBRIDGE
}

\section{Introduction}

Let $M_{n}(k)$ denote the algebra of $n \times n$ matrices over some field $k$ of characteristic zero. For each $k$-valued function $\chi$ on the symmetric group $S_{n}$, we may define a corresponding matrix function on $M_{n}(k)$ in which

$$
\left(a_{i j}\right) \longmapsto \sum_{w \in S_{n}} \chi\left(w^{-1}\right) a_{1, w(1)} \ldots a_{n, w(n)} .
$$

If $\chi$ is an irreducible character of $S_{n}$, these functions are known as immanants; if $\chi$ is an irreducible character of some subgroup $G$ of $S_{n}$ (extended trivially to all of $S_{n}$ by defining $\chi(w)=0$ for $w \notin G)$, these are known as generalized matrix functions. Note that the determinant and permanent are obtained by choosing $\chi$ to be the sign character and trivial character of $S_{n}$, respectively.

We should point out that it is more traditional to use $\chi(w)$ in (1) where we have used $\chi\left(w^{-1}\right)$. This change can be undone by transposing the matrix. If $\chi$ happens to be a character, then $\chi\left(w^{-1}\right)=\bar{\chi}(w)$, so the generalized matrix function we have indexed by $\chi$ is the complex conjugate of the traditional one. Since the characters of $S_{n}$ are real (and integral), it follows that there is no difference between our indexing of immanants and the traditional one.

It is convenient to associate with each $A \in M_{n}(k)$ the following element of the group algebra $k S_{n}$ :

$$
[A]:=\sum_{w \in S_{n}} a_{1, w(1)} \ldots a_{n, w(n)} \cdot w^{-1} .
$$

In terms of this notation, the matrix function defined by (1) can be described more simply as $A \mapsto \chi[A]$, provided that we extend $\chi$ linearly to $k S_{n}$. Note that if we had used $w$ in place of $w^{-1}$ here and in (1), then the restriction of [.] to the group of permutation matrices would have been an anti-homomorphism, rather than a homomorphism.

Recall that a (real) matrix $A$ is totally positive if all minors of $A$ are nonnegative (that is, every submatrix has nonnegative determinant). We shall use TP as an abbreviation for the set of totally positive $A \in M_{n}(\mathbf{R})$. In a previous paper [11], we developed a series of conjectures for immanants, the most fundamental of which was the conjecture that immanants of totally positive matrices are nonnegative. In this paper, we shall prove this conjecture, as well as one of the other conjectures in [11] concerning the convex cone in $\mathbf{R} S_{n}$ spanned by the elements $[A]$ for $A \in \mathrm{TP}$.

Received 25 January 1991.

1980 Mathematics Subject Classification 15A15, 20C30, 15 A48.

Research partially supported by NSF Grants DMS-8807279 and DMS-9057192.

Bull. London Math. Soc. 23 (1991) 422-428 
Let $\operatorname{deg}(\chi)=\chi$ (id) denote the degree of any character $\chi$. One of the consequences of our proof of the above conjectures will be the following evidently stronger result for immanants:

$$
\chi[A] \geqslant \operatorname{deg}(\chi) \operatorname{det}(A) \quad(A \in \mathrm{TP}) .
$$

This is analogous to Schur's Dominance Theorem [10], which asserts that the same inequality is true for generalized matrix functions of positive semi-definite Hermitian matrices.

\section{Cauchy-Binet and the group algebra of $S_{n}$}

Although the map [ $\cdot]: M_{n}(k) \rightarrow k S_{n}$ is not a homomorphism of algebras, our first step is to show that there does exist a useful way to describe the effect of $[\cdot]$ on a matrix product. This description can be viewed as a refinement of the Cauchy-Binet formula, which plays an important role in the analysis of total positivity [7]. The existence of this formula was suggested by previous extensions of the Cauchy-Binet formula to generalized matrix functions associated with linear characters by Marcus and Minc [9], and to multivariate kernels by Karlin and Rinott [8].

First, we need to consider submatrices of a given matrix $A$ that are obtained by selecting certain rows and columns, with repetitions allowed. We shall refer to these as generalized submatrices of $A$. More explicitly, let us define

$$
\mathscr{M}_{r}(n)=\left\{\left(i_{1}, \ldots, i_{r}\right) \in \mathbf{Z}^{r}: 1 \leqslant i_{1} \leqslant \ldots \leqslant i_{r} \leqslant n\right\},
$$

and for any such $I \in \mathscr{M}_{r}(n)$, let

$$
z_{I}=\prod_{1 \leqslant i \leqslant n} m_{i} !
$$

where $m_{i}$ denotes the multiplicity of $i$ in $I$. If $A$ is an $m \times n$ matrix and $I \in \mathscr{M}_{r}(m)$, $J \in \mathscr{M}_{s}(n)$, let us define $A_{I, J}$ to be the $r \times s$ (generalized) submatrix obtained by selecting the rows of $A$ indexed by $I$ and columns indexed by $J$. For the special case in which $I$ indexes one copy of each row of $A$, we shall write $A_{*, J}$ for $A_{I, J}$. The notation $A_{I, *}$ will be used analogously.

THEOREM 1.1. Let $A$ and $B$ be matrices of size $p \times q$ and $q \times r$, respectively. If $I \in \mathscr{M}_{n}(p), J \in \mathscr{M}_{n}(r)$ and $C=A B$, then

$$
\left[C_{I, J}\right]=\sum_{K \in \mathscr{M}_{n}(q)} \frac{1}{z_{K}}\left[A_{I, K}\right] \cdot\left[B_{K, J}\right] .
$$

To recover the Cauchy-Binet identity, one merely applies the sign character of $S_{n}$ to (2); each occurrence of $[\cdot]$ becomes a determinant, and the only possibly nonzero terms in the sum correspond to $n$-tuples $K$ without repetition. More generally, if one applies a linear character of any subgroup of $S_{n}$ to (2), one recovers the generalized Cauchy-Binet identity due to Marcus and Minc [9].

Proof. Let $A^{\prime}=A_{I, *}$ and $B^{\prime}=B_{*, J}$, so that $A^{\prime} B^{\prime}=C_{I, J}$. For simplicity, we write $a_{i j}$ and $b_{i j}$ for the $i, j$-entries of $A^{\prime}$ and $B^{\prime}$ (rather than $A$ and $B$ ). In these terms, we have

$$
\begin{aligned}
{\left[A^{\prime} B^{\prime}\right] } & =\sum_{w \in S_{n} i_{1}, \ldots, i_{n}-1} \sum_{1, i_{1}}^{q} b_{i_{1}, w(1)} \ldots a_{n, i_{n}} b_{i_{n}, w(n)} \cdot w^{-1} \\
& =\sum_{w \in S_{n} i_{1}, \ldots, i_{n}-1} \sum_{w}^{q}\left(i_{1}, \ldots, i_{n}\right) w^{-1}
\end{aligned}
$$


where

$$
f_{w}\left(i_{1}, \ldots, i_{n}\right)=a_{1, i_{1}} \ldots a_{n, i_{n}} b_{i_{1}, w(1)} \ldots b_{i_{n}, w(n)} .
$$

In general, if $f$ is any function of $n$ indices, one has

$$
\sum_{i_{1}, \ldots, i_{n}-1}^{q} f\left(i_{1}, \ldots, i_{n}\right)=\sum_{K \in \mathscr{M}_{n}(q)} \frac{1}{z_{K}} \sum_{w \in S_{n}} f\left(k_{w(1)}, \ldots, k_{w(n)}\right),
$$

where $K=\left(k_{1}, \ldots, k_{n}\right)$, since each distinct rearrangement of $\left(k_{1}, \ldots, k_{n}\right)$ is realized by exactly $z_{K}$ different permutations $w$.

Applying this reasoning to (3), we obtain

$$
\begin{aligned}
{\left[A^{\prime} B^{\prime}\right] } & =\sum_{K \in \mathscr{M}_{n}(q)} \frac{1}{z_{K}} \sum_{w, w^{\prime} \in S_{n}} a_{1, k_{w(1)}} \ldots a_{n, k_{w(n)}} b_{k_{w(1)}, w^{\prime}(1)} \ldots b_{k_{w(n)}, w^{\prime}(n)} \cdot w^{\prime-1} \\
& =\sum_{K \in \mathscr{M}_{n}(q)} \frac{1}{z_{K}} \sum_{w, w^{\prime} \in S_{n}} a_{1, k_{w(1)}} \ldots a_{n, k_{w(n)}} b_{k_{1}, w^{\prime} w^{-1}(1)} \ldots b_{k_{n}, w^{\prime} w^{-1}(n)} \cdot w^{\prime-1} \\
& =\sum_{K \in \mathscr{M}_{n}(q)} \frac{1}{z_{K}} \sum_{w, w^{*} \in S_{n}} a_{1, k_{w(1)}} \ldots a_{n, k_{w(n)}} b_{k_{1}, w^{*}(1)} \ldots b_{k_{n}, w^{*}(n)} \cdot\left(w^{\prime \prime} w\right)^{-1} \\
& =\sum_{K \in \mathscr{M}_{n}(q)} \frac{1}{z_{K}}\left[A_{*, K}^{\prime}\right]\left[B_{K, *}^{\prime}\right] .
\end{aligned}
$$

Since $A_{*, K}^{\prime}=A_{I, K}$ and $B_{K, *}^{\prime}=B_{K, J}$, this agrees with (2).

Let $\rho: \mathbf{R} S_{n} \rightarrow \mathrm{GL}_{m}(\mathbf{R})$ be any matrix representation of $\mathbf{R} S_{n}$. We shall say that $A \in M_{n}(\mathbf{R})$ is $\rho$-positive if the matrix entries of $\rho([A])$ are nonnegative. For such $A$, one clearly has $\chi[A] \geqslant 0$, where $\chi$ denotes the character of $\rho$.

Let us also define $A \in M_{n}(\mathbf{R})$ to be strictly $\rho$-positive if $A$ and also all of its $n \times n$ generalized submatrices are $\rho$-positive. An immediate consequence of Theorem 1.1 is the following.

COROLlaRy 1.2. If $A$ and $B$ are strictly $\rho$-positive, then so is $A B$.

\section{The cone of total positivity}

For any $S \subset \mathbf{R} S_{n}$, let $\mathscr{C}(S)$ denote the convex cone spanned by nonnegative linear combinations of elements of $S$. If $\mathscr{C}(S)$ is closed under the product in $\mathbf{R} S_{n}$, we shall say that $\mathscr{C}(S)$ is multiplicative. Let $\mathscr{C}^{*}(S)$ denote the smallest multiplicative cone containing $S$; clearly, $\mathscr{C}^{*}(S)=\mathscr{C}\left(S^{*}\right)$, where $S^{*}$ denotes the set of products of elements of $S$, including $S$ itself.

The cone we are interested is the one generated by $S=\{[A]: A \in \mathrm{TP}\}$; let us denote this 'cone of total positivity' by $\mathscr{C}_{\mathrm{TP}}$. Although the geometric structure of this cone is not well understood (for example, it is not even known whether it is polyhedral), we can show that its multiplicative closure $\mathscr{C}_{\mathrm{TP}}^{*}$ has a fairly simple set of generators.

For any subinterval $[i, j]$ of $\{1,2, \ldots, n\}$, let $x_{[i, j]} \in \mathbf{R} S_{n}$ denote the sum of all permutations $w \in S_{n}$ that fix every $k \notin[i, j]$ (for example, $x_{[2,4]}=\mathrm{id}+(23)+(34)+$ $(24)+(234)+(243))$. Define

$$
\Sigma=\{\text { id }\} \cup\left\{x_{[i, j]}: 1 \leqslant i<j \leqslant n\right\} .
$$


The following result confirms Conjecture 5.3 of [11].

THEOREM 2.1. $\mathscr{C}_{\mathrm{TP}}^{*}=\mathscr{C}^{*}(\Sigma)$. In particular, the cone of total positivity is contained in the multiplicative cone generated by $\Sigma$.

Our proof depends crucially on the following theorem of Cryer [2] (compare also Theorem 3.5 and Corollary 3.6 of [1]).

THEOREM 2.2. Assume that $A \in M_{n}(\mathbf{R})$ is totally positive.

(a) There is a factorization $A=L U$, with $L$ and $U$ lower and upper triangular (respectively), in which $L$ and $U$ are totally positive.

(b) If $A$ is upper triangular, then there is a factorization $A=U_{1} \ldots U_{l}$ in which each $U_{i}$ is upper triangular, tri-diagonal, and entrywise nonnegative.

It is easy to check that any upper triangular matrix $U=\left[u_{i j}\right]$ that satisfies the conditions in part (b) (that is, $u_{i j} \geqslant 0$ for $j-i=0,1$; and $u_{i j}=0$ otherwise) is totally positive.

LEMMA 2.3. If $U \in M_{n}(\mathbf{R})$ is upper triangular, tri-diagonal and nonnegative, then $\left[U_{I, J}\right] \in \mathscr{C}^{*}(\Sigma)$ for all $I, J \in \mathscr{M}_{n}(n)$.

Proof. Let $r_{i}$ (respectively $s_{i}$ ) denote the multiplicity of $i$ in $I$ (respectively $J$ ). We may partition the rows (respectively columns) of $U_{I, J}$ into blocks of size $r_{1}, \ldots, r_{n}$ (respectively $s_{1}, \ldots, s_{n}$ ) so that each block-submatrix of $U_{I, J}$ has constant entries. For example, if $n=4$, we have

$$
U_{I, J}=\left[\begin{array}{cccc}
u_{11} E_{r_{1}, s_{1}} & u_{12} E_{r_{1}, s_{2}} & 0 & 0 \\
0 & u_{22} E_{r_{2}, s_{2}} & u_{23} E_{r_{2}, s_{3}} & 0 \\
0 & 0 & u_{33} E_{r_{3}, s_{3}} & u_{34} E_{r_{3}, s_{4}} \\
0 & 0 & 0 & u_{44} E_{r_{4}, s_{4}}
\end{array}\right],
$$

where $E_{r, s}$ denotes the $r \times s$ matrix of ones.

Let $Z$ denote the special instance of $U$ obtained by setting $u_{11}=u_{12}=u_{22}=$ $\ldots=1$, and define $\mathscr{S}_{I, J}$ to be the set of $w \in S_{n}$ such that the coefficient of $w$ in $\left[Z_{I, J}\right]$ is nonzero. We shall assume that $\mathscr{S}_{I, J}$ is nonempty, since otherwise we have $\left[U_{I, J}\right]=\left[Z_{I, J}\right]=0$.

Given that $\mathscr{S}_{I, J}$ is nonempty, it must be possible to place $n$ non-attacking rooks on the set of nonzero matrix entries of $Z_{1, J}$. In particular, this requires placing $s_{1}$ rooks on the $r_{1} \times s_{1}$ block, $r_{1}-s_{1}$ rooks on the $r_{1} \times s_{2}$ block, $s_{2}-r_{1}+s_{1}$ rooks on the $r_{2} \times s_{2}$ block, and so on. We therefore have

$$
s_{i+1} \geqslant r_{i}-s_{i}+\ldots+r_{1}-s_{1} \geqslant 0 \quad(i \geqslant 1) .
$$

These inequalities imply that $\mathscr{S}_{I, J}$ contains the identity permutation.

Let $S_{I}$ denote the subgroup of $S_{n}$ that fixes $I$. For any $w \in S_{I}$, we have $w \mathscr{S}_{I, J}=$ $\mathscr{S}_{I, J}$ and $w\left[U_{I, J}\right]=\left[U_{I, J}\right]$. Similarly, if $w \in S_{J}$ then $\mathscr{S}_{I, J} w=\mathscr{S}_{I, J}$ and $\left[U_{I, J}\right] w=$ $\left[U_{I, J}\right]$. A moment of thought shows that the combination of these left and right actions is transitive on $\mathscr{S}_{I, J}$, so we have

$$
\mathscr{S}_{I, J}=S_{I} \cdot S_{J}
$$


This also shows that the number of ways to express a fixed $w \in \mathscr{S}_{I, J}$ in the form $w_{1} \cdot w_{2}$ with $w_{1} \in S_{I}$ and $w_{2} \in S_{J}$ does not depend on $w$. If we choose $w=$ id, we see that this number is $\left|S_{I} \cap S_{J}\right|$. Using $u_{I, J}$ to denote the product of the diagonal entries of $U_{I, J}$ (that is, the coefficient of id in $\left.\left[U_{I, J}\right]\right)$, it follows that

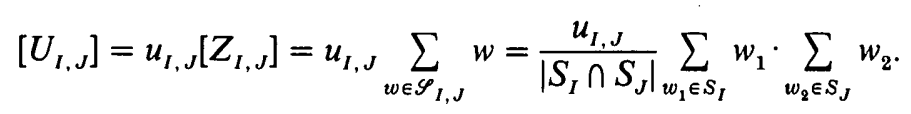

However,

$$
\sum_{w_{1} \in S_{I}} w_{1}=x_{\left[1, r_{1}\right]} x_{\left[r_{1}+1, r_{1}+r_{2}\right]} \ldots x_{\left[r_{1}+\ldots+r_{n-1}+1, n\right]},
$$

and there is a similar factorization for $S_{J}$. Hence $\left[U_{I, J}\right]$ is a nonnegative multiple of products of elements of $\Sigma$, and thus a member of $\mathscr{C}^{*}(\Sigma)$.

Proof of Theorem 2.1. Let $I, J \in \mathscr{M}_{n}(n)$. If $U \in \mathrm{TP}$ is upper triangular, then Theorems 2.2(b) and 1.1 show that $\left[U_{I, J}\right]$ is a nonnegative linear combination of products of terms of the form $\left[U_{K, L}^{\prime}\right]$ for various upper triangular, tri-diagonal, nonnegative matrices $U^{\prime}$. By Lemma 2.3 , these terms belong to $\mathscr{C}^{*}(\Sigma)$, so we conclude that $\left[U_{I, J}\right] \in \mathscr{C}^{*}(\Sigma)$ for all upper triangular $U \in \mathrm{TP}$.

Next, let ${ }^{\dagger}$ denote the anti-automorphism of $\mathbf{R} S_{n}$ defined by $w \mapsto w^{-1}$ (extended linearly). Note that $x_{[i, j]}^{\dagger}=x_{[i, j]}$, so $\mathscr{C}^{*}(\Sigma)$ is ${ }^{\dagger}$-invariant. Since $[A]^{\dagger}=\left[A^{t}\right]$, it follows that $\left[L_{I, J}\right] \in \mathscr{C}^{*}(\Sigma)$ for all lower triangular $L \in \mathrm{TP}$.

By Theorem 2.2(a) and a second application of Theorem 1.1, we may conclude that $[A] \in \mathscr{C}^{*}(\Sigma)$ for all $A \in \mathrm{TP}$; that is, $\mathscr{C}_{\mathrm{TP}}^{*} \subseteq \mathscr{C}^{*}(\Sigma)$. Conversely, one knows $\mathscr{\mathscr { C }}_{\mathrm{TP}}^{*} \supseteq$ $\mathscr{C}^{*}(\Sigma)$, since each generator $x_{[i, j]}$ of $\mathscr{C}^{*}(\Sigma)$ is of the form $[A]$ for suitable $A \in \mathrm{TP}$. (Take $A$ to be the 0-1 matrix with 1s on the diagonal and in the rows and columns of $[i, j]$.)

\section{The dénouement}

To prove that immanants of totally positive matrices are nonnegative, we shall show, in fact, that for every irreducible character $\chi$ of $S_{n}$, there exists a matrix representation $\rho$ of $\mathbf{R} S_{n}$ with character $\chi$ such that $A$ is $\rho$-positive for every $A \in \mathrm{TP}$.

For this, we may rely on the following theorem of Greene [4].

THEOREM 3.1. For each irreducible representation of $\mathbf{R} S_{n}$, the representing matrix for every $x_{[i, j]}$ is nonnegative with respect to the Young seminormal basis.

It should be noted that Greene proved more than just the nonnegativity; he also gave an explicit description of the representing matrices for each $x_{[i, j]}$. Haiman has also pointed out that Theorem 3.1 remains true if one replaces the Young seminormal basis with the Kazhdan-Lusztig basis [5].

In view of Theorem 2.1, we may now deduce the following.

THEOREM 3.2. If $A \in M_{n}(\mathbf{R})$ is totally positive and $\rho$ is an irreducible representation of $\mathbf{R} S_{n}$, then the matrix entries of $\rho([A])$ are nonnegative with respect to the Young seminormal basis (as well as the Kazhdan-Lusztig basis) for $\rho$.

Taking traces, we obtain Conjecture 2.2 of [11]. 
COROLlary 3.3. Immanants of totally positive matrices are nonnegative, that is, $\chi[A] \geqslant 0$ for every $A \in \mathrm{TP}$ and every character $\chi$ of $S_{n}$.

With slightly more work, we can prove the analogue of Schur's Dominance Theorem mentioned in the Introduction.

Corollary 3.4. If $A \in \mathrm{TP}$ and $\chi$ is a character of $S_{n}$, then

$$
\chi[A] \geqslant \operatorname{deg}(\chi) \operatorname{det}(A) .
$$

Proof. Let sgn: $\mathbf{R} S_{n} \rightarrow \mathbf{R}$ denote the sign homomorphism. The claimed inequality is equivalent to

$$
\chi(c)-\operatorname{deg}(\alpha) \operatorname{sgn}(c) \geqslant 0
$$

for all $c \in \mathscr{C}_{\mathrm{TP}}$. Since Theorem 2.1 shows that $\mathscr{C}_{\mathrm{TP}} \subseteq \mathscr{C}^{*}(\Sigma)$, it suffices to prove (4) whenever $c$ is a product of generators in $\Sigma$. If $c=$ id, then equality occurs. Otherwise, if $c$ is a product of one or more of the generators $x_{[i, j]}$, then the fact that $\operatorname{sgn}\left(x_{[i, j]}\right)=0$ shows that the nonnegativity of (4) is equivalent to $\chi(c) \geqslant 0$. This is a consequence of Theorem 3.1 .

Since Schur's Dominance Theorem applies not only to immanants but also to generalized matrix functions, it is natural to ask whether Corollary 3.4 can be extended to this more general case. In order to make this question meaningful, it is reasonable to restrict our attention to characters of subgroups of $S_{n}$ that are realvalued. Without this restriction, we could not guarantee that $\chi[A]$ would be realvalued for all $A \in \mathrm{TP}$. The following example, due to Gordon James [6], shows that even under these circumstances, generalized matrix functions of totally positive matrices need not be nonnegative.

Let $G$ be the subgroup of $S_{6}$ generated by $(1,2)(4,5)$ and $(2,3)(5,6)$. Note that $G \cong S_{3}$. Let $\chi$ be the sign character of $G$, so that $\chi((1,2)(4,5))=\chi((2,3)(5,6))=-1$. For any $A, B \in M_{3}(\mathbf{R})$, we have

$$
\chi[A \oplus B]=\operatorname{det}(A \circ B),
$$

where $A \circ B$ denotes the Hadamard (that is, entrywise) product of $A$ and $B$. If we take

$$
A=B^{t}=\left[\begin{array}{lll}
1 & 1 & 1 \\
1 & 1 & 1 \\
0 & 1 & 1
\end{array}\right],
$$

then $A \oplus B$ is totally positive but $\operatorname{det}(A \circ B)=-1$.

As a final remark, it is interesting to point out that one of the stronger conjectures that remains open (namely, Conjecture 2.1 of [11]) would characterize the convex cone of linear functionals $\phi: \mathbf{R} S_{n} \rightarrow \mathbf{R}$ that are constant on the conjugacy classes of $S_{n}$ and nonnegative on the cone of total positivity. In view of Theorem 2.1, it would suffice to prove that these functionals are also nonnegative on the cone $\mathscr{C}^{*}(\Sigma)$; this is the content of Conjecture 5.2 in [11].

ACKNOWLEDGEMENTS. I should like to extend thanks to Charles R. Johnson for bringing the paper of Ando to my attention, and also to Ian Goulden and David Jackson for their paper [3], which aroused my interest in immanants. 


\section{References}

1. T. ANDo, 'Totally positive matrices', Linear Algebra Appl. 90 (1987) 165-219.

2. C.W. CRYER, 'Some properties of totally positive matrices', Linear Algebra Appl. 15 (1976) 1-25.

3. I. P. GOULDEN and D. M. JACKSON, 'Immanants of combinatorial matrices', J. Algebra, to appear.

4. C. GreENE, 'Proof of a conjecture on immanants of the Jacobi-Trudi matrix', Linear Algebra Appl., to appear.

5. M. D. Haiman, 'Immanant conjectures and Kazhdan-Lusztig polynomials', preprint.

6. G. D. JAMES, private communication.

7. S. KaRLIN, Total positivity (Stanford University Press, 1968).

8. S. KARLIN and Y. RinOTT, 'A generalized Cauchy-Binet formula and applications to total positivity and majorization', J. Multivariate Anal. 27 (1988) 284-299.

9. M. Marcus and H. MiNC, 'Generalized matrix functions', Trans. Amer. Math. Soc. 116 (1965) 316-329.

10. I. SCHUR, 'Über endliche Gruppen und Hermitesche Formen', Math. Z. 1 (1918) 184-207.

11. J. R. STEMBRIDGE, 'Some conjectures for immanants', Canad. J. Math., to appear.

Department of Mathematics

University of Michigan

Ann Arbor

Michigan 48109-1003

USA 